\title{
Cryptococcal meningitis as a complication of systemic lupus erythematosus treated with systemic corticosteroids
}

\author{
J. V. Collins \\ M.B., M.R.C.P.
}

\author{
D. ToNG \\ B.Sc., M.B., B.S.
}

Guys Hospital, London, S.E. 1

\author{
R. G. BUCKNALL \\ M.B., B.S. \\ Guy's Hospital London, S.E.1
}

\author{
A. P. WARIN \\ M.B., M.R.C.P.
}

St Nicholas' Hospital, Plumstead, London, S.E.18

\section{Summary}

Cryptococcosis, a disease of world-wide distribution, is uncommon in the British Isles where it usually presents as meningitis affecting patients with malignant disease of the reticulo-endothelial system, diabetes mellitus or those receiving systemic treatment with corticosteroids. The clinical features and physical signs of the disease are often non-specific and resemble those of tuberculous meningitis. The diagnosis is made upon recognizing the encapsulated Cryptococcus neoformans on Indian Ink preparations of the cerebrospinal fluid, or by demonstrating precipitating antibodies to the organism in the patient's serum. The treatment is by intravenous administration of amphotericin B for a period of 6-12 weeks, supplemented in severe or relapsing cases by intrathecal therapy; alternatively, 5-fluorocytosine may be given by mouth. A case is reported of cryptococcal meningitis occurring in a 46-year-old woman with systemic lupus erythematosus treated with methylprednisolone. Treatment with amphotericin B was successful in suppressing the infection, but was accompanied by side-effects and some temporary impairment of renal function.

\section{Introduction}

Patients who are treated with immunosuppressive drugs show a higher incidence of all types of infections than the general population, but of particular interest is their increased susceptibility to less common infections such as the mycoses. We report the occurrence of cryptococcal meningitis in a woman receiving long-term corticosteroid treatment for systemic lupus erythematosus.

\section{Case report}

A 46-year-old female shorthand typist was admitted to St Olave's Hospital (Guy's Group), on 22 September 1970, with a 3-week history of duli frontal headache, malaise and low-grade fever Three years previously, she had presented with $a ?$ respiratory illness and polyarthritis, and a diagnosis of systemic lupus erythematosus was made and confirmed by laboratory findings of positive ANF, abundant LE cells, positive latex flocculation and positive Rose-Waaler to a titre of $1 / 24$. Treatment was begun with systemic corticosteroids. Since that time, she had remained symptomless on $10-15 \mathrm{mg}$ methylprednisolone daily.

On the present admission (22 September 1970) she was confused and drowsy, but free of headache and other symptoms. She had a sinus tachycardia of $130 /$ min, but no other abnormal physical signs. The chest radiograph was normal. Later that day, she became febrile $\left(39.7^{\circ} \mathrm{C}\right.$ ) and was noted to have oral candidiasis. Twenty-four hours after admission, her level of consciousness had fallen but the only focal physical sign was an extensor plantar response on the left. A lumbar puncture produced clear cerebrospinal fluid under $230 \mathrm{~mm} \mathrm{H}_{2} \mathrm{O}$ pressure, containing protein $150 \mathrm{mg} / 100 \mathrm{ml}$, WBC $40 / \mathrm{mm}^{3}$ (92\% neutrophils). A Gram stain showed scanty yeast cells, which on an Indian Ink preparation were confirmed as encapsulated Cryptococcus neoformans, later confirmed by culture.

Treatment was begun on 23 September 1970 by changing to oral prednisolone $60 \mathrm{mg}$ daily and intravenous treatment with amphotericin B was commenced with an initial dose of $1 \mathrm{mg}$ in $250 \mathrm{ml}$ 
of $5 \%$ dextrose solution infused over $6 \mathrm{hr}$. In addition, she was given $0.5 \mathrm{mg}$ amphotericin B mixed with $5 \mathrm{ml}$ of CSF and $20 \mathrm{mg}$ hydrocortisone hemisuccinate by slow intrathecal injection into the lumbar subarachnoid space.

No side-effects were encountered, and by the fourth day of therapy (26 September 1970) the full parenteral dose of $30 \mathrm{mg}$ amphotericin $B(0.75 \mathrm{mg} /$ $\mathrm{kg}$ body weight) was reached. This dose was subsequently infused each day in 11 of $5 \%$ dextrose solution containing 500 units of heparin over a period of 6-8 hr, using a scalp vein canula into a forearm vein. The canula was removed at the end of each infusion and the infusion site was changed regularly. No local complications of this technique were encountered. Daily intravenous amphotericin B was given for 6 weeks with a maintenance dose of oral prednisolone $30 \mathrm{mg}$ daily. Intrathecal amphotericin $B, 0.5 \mathrm{mg} /$ dose, was given in the manner described above, 2 days each week for 5 weeks. Her fever had resolved within 4 days of starting treatment and she became well orientated soon after. During the fourth week of treatment she became febrile $\left(37.7^{\circ} \mathrm{C}\right)$ and had clinical signs of a deep vein thrombosis of the right calf which was treated by bandaging and elevation of the limb.

A full blood picture, blood urea and electrolyte estimations were performed twice weekly and the urine was examined daily for blood and protein. In addition, creatinine clearance studies were performed at regular intervals. At each lumbar puncture, Cryptococcus neoformans could still be identified in the cerebrospinal fluid when this was centrifuged, but the organism failed to grow on culture. After 5 weeks of treatment, lumbar puncture was becoming difficult due to fibrosis, and intrathecal treatment was stopped. Because of the persistence of Cryptococcus in the cerebrospinal fluid, the intravenous dose was raised to $40 \mathrm{mg}$ daily $(1 \mathrm{mg} / \mathrm{kg}$ body weight) but this was followed by the development of headache, vomiting and nausea, with fever $\left(37 \cdot 7^{\circ} \mathrm{C}\right)$ without change in physical signs. Amphotericin B was discontinued for $48 \mathrm{hr}$, but no symptomatic improvement occurred and daily intravenous treatment was re-started with a daily dose of $40 \mathrm{mg}$ amphotericin B. The vomiting and fever resolved within 5 days.

Treatment was discontinued after 12 weeks and the patient was allowed home for Christmas, returning for a further 3 weeks treatment with intravenous amphotericin B. At the end of this period, she was symptomless and afebrile. Attempts at lumbar puncture resulted in 'dry taps' and spinal root irritation, and these were therefore abandoned. She had been given a total of $3.8 \mathrm{~g}$ amphotericin B by intravenous infusion and $6.5 \mathrm{mg}$ by intrathecal injection. The patient was allowed home on prednisolone $20 \mathrm{mg}$ daily and 40 weeks after the end of treatment she remains well.

\section{Discussion}

Sporadic infections caused by Cryptococcus neoformans occur throughout the world, and in parts of North America and elsewhere cryptococcosis is endemic. However, in the United Kingdom, the disease is rare, and following the first report of the disease in Britain (Smith \& Crawford, 1930), fewer than fifty cases have appeared in the literature, although there have probably been unreported cases (Symmers, 1967). The source of such infections

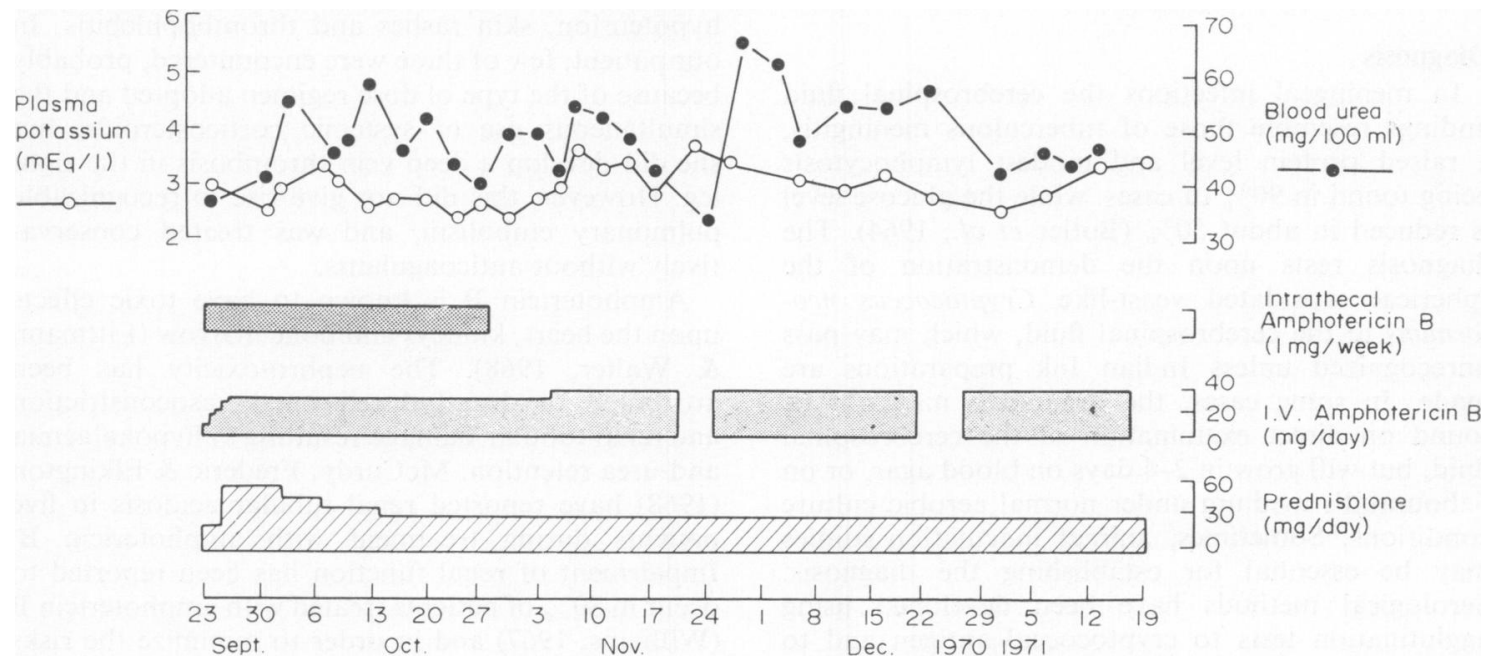

FIG. 1. Details of treatment and changes in blood urea and serum potassium concentrations in a case of cryptococcal meningitis. 
remained unrecognized until Emmons (1955) isolated the yeast-like organism from pigeon droppings, and subsequent studies have demonstrated its presence in pigeon excreta in various parts of Britain (Randhawa, Clayton \& Riddell, 1965; Partridge \& Winner, 1966).

In regions where the disease is endemic, infections are commoner in men, with two-thirds of cases occurring between the third and fifth decades. The disease is prevalent in patients with Hodgkin's disease, lymphosarcoma, and diabetes mellitus; in some series, $50 \%$ of the cases occurred in association with such underlying diseases (Wilson et al., 1970). Where the disease is endemic, the primary route of infection is the lungs, although one-third of such pulmonary infections are without symptoms, and diagnosis is made as an incidental finding on a chest radiograph. In Britain, the disease usually presents as an infection of the nervous system with meningitis being by far the commonest manifestation. As in the case reported here, it is of insidious onset, with an ill-defined headache, often intermittent at first. Later, the patient may show depression, disorientation, confusion and focal neurological signs. The occurrence of these features in a patient with a reticulosis or one who is receiving immunosuppressive drugs should alert the clinician to the possibility of meningeal infection. Advanced infection will give rise to overt signs of meningeal involvement with neck stiffness and a positive Kernig's sign. Localized cryptococcal granulomata in the brain or spinal cord may mimic tumour, abscess or subdural haematomata. Cryptococcal infections may frequently resemble the more familiar tuberculosis of the nervous system and may present similar difficulties in diagnosis.

\section{Diagnosis}

In meningeal infections the cerebrospinal fluid findings resemble those of tuberculous meningitis, a raised protein level and modest lymphocytosis being found in $90 \%$ of cases, while the glucose level is reduced in about 50\% (Butler et al., 1964). The diagnosis rests upon the demonstration of the spherical capsulated yeast-like Cryptococcus neoformans in the cerebrospinal fluid, which may pass unrecognized unless Indian Ink preparations are made. In some cases, the organisms may not be found on direct examination of the cerebrospinal fluid, but will grow in 2-4 days on blood agar, or on Sabouraud's medium under normal aerobic culture conditions. Sometimes, animal inoculation studies may be essential for establishing the diagnosis. Serological methods have been developed using agglutination tests to cryptococcal antigen and to suspensions of Cryptococcus neoformans (Gordon $\&$ Vedder, 1966). These tests are of particular value in establishing the diagnosis in those cases where the organism has not been isolated or grown on culture.

\section{Treatment}

Until the discovery of amphotericin B by Gold et al. in 1956, cryptococcal meningitis was a fatal disease, $90 \%$ of cases dying within 1 year of infection. Treatment with this drug has been successful in $70-80 \%$ of patients, but subsequent relapse may occur in $30 \%$ (Butler et al., 1964). It is thought that amphotericin B renders the cryptococcus susceptible to the natural defences of the body without destroying the organism, and for this reason administration for a long period on low dosage is thought to be more effective than trying to achieve very high blood levels. Amphotericin B is given as an intravenous infusion in $5 \%$ dextrose solution in water, over a period of $6 \mathrm{hr}$ daily for 6-12 weeks until a total dose of at least $3 \mathrm{~g}$ has been given (Williams, 1967). Treatment is begun with $1 \mathrm{mg}$ of amphotericin B in $250 \mathrm{ml}$ of $5 \%$ dextrose solution and thereafter the dose is increased by 5 or $10 \mathrm{mg}$ daily until the full daily dose of $1 \mathrm{mg} / \mathrm{kg}$ body weight is reached. In resistant or severe cases, additional intrathecal amphotericin $B$ is given by mixing $0.25 \mathrm{mg} t \& \overrightarrow{0}$ $0.5 \mathrm{mg}$ of drug with $5 \mathrm{ml}$ cerebrospinal fluid an $15-20 \mathrm{mg}$ hydrocortisone hemisuccinate. This mixê. ture is given by slow injection into the lumba subarachnoid space. This procedure is repeated for 2-3 days each week up to a maximum total intrathecal dose of $15 \mathrm{mg}$ amphotericin B.

Treatment with amphotericin B may be accompanied by a multiplicity of side-effects which may on occasion prevent its continuance. These include nausea and vomiting, rigors, muscle pains, tremors, hypotension, skin rashes and thrombophlebitis. In our patient, few of these were encountered, probably because of the type of dose regimen adopted and the simultaneous use of systemic corticosteroids, but she did develop a deep vein thrombosis in the right leg. However, this did not give rise to recognizable pulmonary embolism, and was treated conservatively without anticoagulants.

Amphotericin B is known to have toxic effects upon the heart, kidneys and bone marrow (Littmann \& Walter, 1968). The nephrotoxicity has been attributed to drug-induced renal vasoconstriction and renal tubular damage resulting in hypokalaemia and urea retention. McCurdy, Frederic \& Elkington (1968) have reported renal tubular acidosis in five patients during treatment with amphotericin B. Impairment of renal function has been reported to occur in $80 \%$ of patients treated with amphotericin B (Williams, 1967) and in order to minimize the risks of this and other toxic effects, various dose regimens have been employed. Drutz et al. (1968) have argued 
that effective treatment of cryptococcosis will ensue if a maximum serum concentration of amphotericin B equivalent to twice the minimum in vitro inhibitory concentration for Cryptococcus neoformans is achieved during the daily infusion. Based upon this, they have reported successful treatment of cryptococcosis using smaller daily doses than the usual $1 \mathrm{mg} / \mathrm{kg}$ body weight. In keeping with their suggestions, we began treatment with a daily intravenous dose of $0.75 \mathrm{mg} / \mathrm{kg}$ body weight, and this resulted in a resolution of symptoms, but our failure to eradicate Cryptococcus neoformans from the cerebrospinal fluid led us to raise the dose to $1.0 \mathrm{mg} / \mathrm{kg}$ body weight after 6 weeks of treatment. The recrudescence of headache, vomiting and fever which followed this change of dcse was thought to be a toxic reaction which resolved after treatment had been restarted and continued for a few days.

Our patient developed hypokalaemia and a rising blood urea, but paradoxically was shown to have a rise in creatinine clearance during the course of treatment. Regular oral potassium supplements were only partly effective in restoring the serum potassium to normal levels and at the end of treatment, the creatinine clearance rate had returned to pre-treatment levels and the blood urea was higher than before treatment.

It has been suggested that systemic corticosteroids may adversely affect the response to treatment by diminishing the effectiveness of the natural defences (Gordon \& Vedder, 1966), but in the present case, it was considered that any change in steroid therapy was likely to result in a recrudescence of systemic lupus erythematosus, and prednisolone was continued in high dosage.

More recently, oral treatment with 5-fluorocytosine has been introduced for the treatment of mycotic infections and fewer side-effects have been reported than with amphotericin B. This is thought to be an anti-metabolite of cytosine in Cryptococcus neoformans and other fungi, and is effective in inhibiting their growth. Success has been reported with this drug in treating crypotococcal meningitis resistant to amphotericin B (Watkins et al., 1969). Ease of administration and freedom from side-effects may make 5-fluorocytosine the first line of treatment in fungal infections, but further reports are awaited before full evaluation can be made. At present, it is probably test reserved for cases which prove resistant to amphotericin B or where treatment with this drug produces excessive side-effects.

The prognosis in cryptococcosis is most favourable when the organisms can be eradicated from the cerebrospinal fluid, but in many cases, as with ours, this may prove impossible and symptomatic cure has to be accepted. Recurrence may occur at any time, but usually occurs within the first year after treatment. A trial of 5-fluorocytosine may be indicated where one full course of amphotericin $B$ has failed.

\section{Acknowledgments}

We wish to thank Dr K. S. MacLean for permission to publish this case and for his helpful criticism of this paper. Similarly, we are indebted to Dr Jane Fullerton for the laboratory investigations and for her encouragement, and to Miss P. Archer, Mr J. Ritina and their assistants in the Departments of Medical Illustration and Photography at Guy's Hospital who were responsible for the figure in the text.

\section{References}

Butler, W.T., Alling, D.W., Spickard, A. \& Utz, J.P. (1964) Diagnostic and prognostic value of clinical and laboratory findings in cryptococcal meningitis. New England Journal of Medicine, 270, 59.

Drutz, D.J., Spickard, A., Rogers, D.E. \& Koenig, M.G. (1968) Treatment of disseminated mycotic infections: A new approach to amphotericin B therapy. American Journal of Medicine, 45, 405.

EMmons, C.W. (1955) Saprophytic sources of Cryptoccus neoformans associated with the pigeon (Columba livia). American Journal of Hygiene, 62, 227.

Gold, W., Stout, H.A., Pagano, J.F. \& Donobick, R. (1956) Amphotericins A and B, antifungal antibiotics produced by a streptomycete. In vitro studies. In: Antibiotics Annual (Ed. by H. Welch \& F. Marti-Ibanez), p. 579. Medical Encyclopedia, Inc., New York.

GoRDON, M.A. \& VEDDER, D.K. (1966) Serologic tests in diagnosis and prognosis of cryptococcosis. Journal of American Medical Association, 197, 961.

LitTMAN, M.L. \& Walter, J.E. (1968) Cryptococcosis: current status. American Journal of Medicine, 45, 222.

McCurdy, D.K., Frederic, M. \& Elkinton, J.R. (1968) Renal tubular acidosis due to amphotericin B. New England Journal of Medicine, 278, 124.

PARTRIDGe, B.M. \& WinNer, H.I. (1966) Cryptococcus neoformans in bird droppings. Lancet, ii, 1251.

Randhawa, H.S., Clayton, Y.M. \& Riddell, R.W. (1965) Isolation of Cryptococcus neoformans from pigeon habitats in London. Nature (London), 208, 801.

Smith, F.B. \& CRAWford, J.S. (1930) Fatal granulomatosis of the central nervous system due to a yeast (Torula). Journal of Pathology and Bacteriology, 33, 291.

Symmers, W.ST.C. (1967) Cryptococcus neoformans in bird droppings. Lancet, i, 159.

Watkins, J.S., Campbell, M.J., Gardner-Medwin, D., Ingham, H.R. \& Murray, I.G. (1969) Two cases of cryptococcal meningitis, one treated with 5-fluorocytosine. British Medical Journal, 3, 29.

Williams, T.W. (1967) Treatment of mycotic meningitis. Modern Treatment, 4, 951.

Wilson, T.S., Fleming, W.A., Robinson, F.L.J. \& Nicholl, B. (1970) Cryptococcal meningitis associated with steroid therapy. Journal of Clinical Pathology, 23, 657. 